\title{
Wharton Jelly Stem Cells inhibits AGS Gastric Cancer Cells through Induction of Apoptosis and Modification of MAPK and NF-KB Signaling Pathways
}

\section{Samaneh Abbasi}

University of Guilan

\section{Reza Bazyar}

Baqiyatallah University of Medical Sciences

Mohammad Ali Saremi

Baqiyatallah University of Medical Sciences

Gholamhoseen Alishiri

Baqiyatallah University of Medical Sciences

Nasrin Seyyedsani

Islamic Azad University

Mahdieh Farhoudi Sefidan Jadid

Islamic Azad University

Afshin Khorrami

Yazd University

\section{Parisa Emami Golmarz}

Islamic Azad University

\section{Gholamreza Jahangirzadeh}

Islamic Azad University

Alireza isazadeh

Tabriz University of Medical Sciences

\section{Saba Hajazimian}

Tabriz University of Medical Sciences

Fatemeh Firouzi Amoodizaj ( $\square$ fatemeh.firoozi96@yahoo.com )

Islamic Azad University

\section{Research Article}

Keywords: Stem cells, Wharton's jelly, Gastric cancer, MAPK, NF-KB

Posted Date: January 18th, 2021 
DOl: https://doi.org/10.21203/rs.3.rs-142543/v1

License: (c) (1) This work is licensed under a Creative Commons Attribution 4.0 International License. Read Full License

Version of Record: A version of this preprint was published at Tissue and Cell on December 1st, 2021. See the published version at https://doi.org/10.1016/j.tice.2021.101597. 


\section{Abstract}

Background and aim: Gastric cancer) GC) is one of the most common cancer with high mortality worldwide. The human Wharton's jelly stem cells (hWJSCs) can inhibit several cancer cells through several molecular pathways. Therefore, the present study aimed to investigate anticancer effects of hWJSCs conditioned medium (hWJSC-CM) and cell-free lysate (hWJSC-CL) against of GC cell line AGS and underlying signaling pathways.

Methods: In this study, we evaluated the effects of hWJSC-CM and hWJSC-CL on viability, proliferation, migration, invasion, apoptosis, and MAPK and NF-KB signaling pathways in AGS cells. Moreover, mRNA expression of genes involved in apoptosis (BAX, BCL2, SMAC, and SURVIVIM), as well as expression of proteins involved in NF-KB and MAPK signaling pathways were evaluated.

Results: The obtained results showed that the hWJSC-CM and hWJSC-CL decreased viability, migration, and invasion of GC cell line AGS in a concentration and time dependent manner. We observed that the hWJSC-CM and hWJSC-CL induced apoptosis pathway through regulation of apoptosis involved genes mRNA expression. In addition, the hWJSC-CM and hWJSC-CL suppressed NF-KB signaling pathways as well as promoted MAPK signaling pathways.

Conclusions: In general, our study suggested that the hWJSC-CM and hWJSC-CL inhibits proliferation and viability of GC cell line AGS through induction of apoptosis, as well as modification of NF-KB and MAPK signaling pathways.

\section{Introduction}

Gastric cancer (GC) is a prevalent cancer and third cause of cancer death worldwide [1]. The prevalence of GC is increasing in the Asian countries and poor early diagnosis cause to lower survival [2]. Nowadays, chemotherapy followed by surgery are the common treatment methods for GC. However, development of drug-resistant in patients with GC decreased efficacy of chemotherapy agents [3]. Thus, use of novel anticancer agents with natural source are required for treatment and prolonged survival of GC patients [4].

Recently, stem cells therapy is an applicable and favorites approach in treatment of various human cancers. The human Wharton's jelly stem cells (hWJSCs) derived from human umbilical cord are an important source of mesenchymal stem cells (MSCs) with significant clinical benefits, which was usually discarded after delivery [5]. The hWJSCs are multipotent with high proliferative potential, and have mesenchymal and embryonic stem cell characteristics [6]. Unlike to other MSCs, the hWJSCs do not cause tumor formation trough immune systems suppression [7]. Moreover, the hWJSCs can be harvested painless in a large population from umbilical cords. The stemness characteristics of hWJSCs maintain for many passages in culture [6, 7]. Previous studies reported that the hWJSCs have an anti-proliferative 
effects against several human cancer cells [8,9]. Moreover, the evidence suggested that the hWJSCs inhibited proliferation of cancer cells through apoptosis induction [10]. However, underlying mechanisms of the hWJSCs anticancer effects have not been identified. Moreover, the effect of hWJSCs has not been evaluated on GC.

Due to the characteristics of MSCs and its anti-cancer effect as well as less side effects of cell therapy than other treatments such as surgery and chemotherapy, the present study investigated the anti-cancer effects of hWJSCs against GC cell line and underlying signaling pathways.

\section{Materials And Methods}

\section{Cell culture}

The human umbilical cords were received from healthy women at full-term delivery time, and the hWJSCs were derived according to our previous study [8]. In addition, the GC cell line AGS was purchased from Cell Bank of Pasteur Institute, Tehran, Iran. Both hWJSCs and AGS cells culture was performed using Roswell Park Memorial Institute (RPMI)-1640 medium containing 10\% fetal bovine serum (FBS) and 1\% penicillin (100 units $/ \mathrm{mL}$ ) and streptomycin $(100 \mu \mathrm{g} / \mathrm{mL})$. The cells incubation was performed at standard condition $\left(37^{\circ} \mathrm{C}\right.$ with $95 \%$ humidity and $\left.5 \% \mathrm{CO}_{2}\right)$.

\section{Preparation of hWJSC-CM and hWJSC-CL}

The conditioned medium and cell lysates were prepared from cultured hWJSCs with 70\% confluence, according to our previous study [8]. Briley, the culture medium was substituted with antibiotic and FBS free medium, and the hWJSCs conditioned medium (hWJSC-CM) was collected after 72 hours. Next, the cultured hWJSCs were washed once with calcium and magnesium free phosphate buffered saline $\left(\right.$ PBS $\left.^{(-)}\right)$, dissociated using trypsin-EDTA, and centrifuged. The obtained cells pellet mixed with the cell lysis buffer with protease inhibitor. Next, cells suspension was centrifuged and supernatant was collected as hWJSCs cell lysate (hWJSC-CL). The both hWJSC-CM and hWJSC-CL were filtersterilized, and the pH were determined, and stored at $-20^{\circ} \mathrm{C}$.

\section{Cancer cells viability assay}

The GC cell line AGS were seeded in a 96-well plates at density of $1.5 \times 10^{4}$ cells/well in $200 \mu$ culture medium, and then incubated for 24 hours. Next, the cancer cells were treated with various concentrations of hWJSC-CM $(38,40,42,44$, and $46 \%)$ and hWJSC-CL $(11,12,13,14$, and 15\%) for 24-72 hours. The treated cancer cells were washed once with PBS, and then $200 \mu \mathrm{L}$ MTT solution $(5 \mathrm{mg} / \mathrm{mL}$ MTT in culture medium) was added to the each well and incubated for 4 hours at standard condition. Next, $50 \mu \mathrm{L}$ dimethyl sulfoxide (DMSO) was added and incubated further for 30 minutes. The optical densities (OD) 
at $570 \mathrm{~nm}$ wavelength were detected using a plate reader for each well. The relative cancer cell viability was calculated as following: cell viability $=\left(\mathrm{OD}_{570}\right.$ of treated samples $/ \mathrm{OD}_{570}$ of untreated samples $) \times 100$.

\section{Wound-healing migration assay}

The cancer cells were seeded in a 6 -well plates at density of $1.5 \times 10^{5}$ cells/well in $2 \mathrm{ml}$ complete culture medium until cells reached $85 \%$ confluence. Next, a wound was produced by scraping with a sterile pipette tip in middle of each well, and detached cells were washed with PBS. The cancer cells were treated with hWJSC-CM (44\%) and hWJSC-CL (14\%), and incubated in standard condition for 48 hours. Finally, the migrated cancer cells were counted by inverted phase-contrast microscope at 0-20 hours.

\section{Transwell invasion assay}

The cancer cells were seeded in upper chamber of a transwell chamber at density of $2 \times 10^{4}$ cells/well in FBS serum-free culture medium and treated with hWJSC-CM (44\%) and hWJSC-CL (14\%). Moreover, complete culture medium supplemented with $10 \%$ FBS was added to lower chamber. After incubation for 24 hours at standard condition, the migrated cancer cells to lower chamber were fixed in $4 \%$ paraformaldehyde for 15 minutes and stained with $0.1 \%$ crystal violet for 10 minutes. Finally, cancer cells in lower chamber were counted in 5 random fields by inverted phase-contrast microscope.

\section{Cancer cells apoptosis assay}

The cancer cells were seeded in a 6-well plates at density of $1.5 \times 10^{5}$ cells/well in $2 \mathrm{ml}$ complete culture medium for 24 hours. The cancer cells were treated with hWJSC-CM (44\%) and hWJSC-CL (14\%), and incubated in standard condition for 48 hours. Next, the cancer cells were dissociated using trypsin-EDTA and resuspended in binding buffer $(400 \mu \mathrm{L})$. Next, the FITC-conjugated Annexin-V $(5 \mu \mathrm{l})$ and propidium iodide (PI) $(10 \mu \mathrm{l})$ were added and incubated at room temperature in dark for 5 minutes. Finally, apoptosis of cancer cells was determined by flow cytometry instrument (Becton Dickinson Bioscience).

\section{Gene expression assay by Real-Time PCR}

The cancer cells were seeded in a 6 -well plates at density of $1.5 \times 10^{5}$ cells/well in $2 \mathrm{ml}$ complete culture medium for 24 hours. The cancer cells were treated with hWJSC-CM (44\%) and hWJSC-CL (14\%), and incubated in standard condition for 48 hours. Next, the cancer cells were dissociated using trypsin-EDTA and resuspended in TRIzol reagent for extraction of total RNA. The complementary DNA (cDNA) was 
synthesized from $2 \mu \mathrm{g}$ total RNA using reverse transcription reagents. The quantitative Real-Time polymerase chain reaction (qRT-PCR) was performed with SYBR green master mix and specific primers of $B A X, B C L 2$, SMAC, and SURVIVIN genes (Table 1). The ACTIN ( $\beta$-actin) gene was used as endogenous control. The calculations were performed by $2^{-\Delta \triangle C t}$ (Livak) formula.

\section{Proteins expression assay by western blotting}

The cancer cells were seeded in a 6 -well plates at density of $1.5 \times 10^{5}$ cells/well in $2 \mathrm{ml}$ complete culture medium for 24 hours. The cancer cells were treated with hWJSC-CM (44\%) and hWJSC-CL (14\%), and incubated in standard condition for 48 hours. Next, the cancer cells were dissociated using trypsin-EDTA, and lysed in ice-cold RIPA lysis buffer containing protease inhibitor. The cell lysates centrifuged and supernatant collected. The protein levels of p-JNK, JNK, p-p38, p38, p-p65, and p65 were evaluated using western blot analysis. The protein content of cells $(\sim 100 \mu \mathrm{g})$ were separated using sodium dodecyl sulfate polyacrylamide gel electrophoresis (SDS-PAGE) followed by transferring to polyvinylidene difluoride (PVDF) membrane. The membrane was blocked using non-fat dry milk (5\%) and then incubated with primary antibodies at $4^{\circ} \mathrm{C}$ for 24 hours. The horseradish peroxidase (HRP) conjugated antibody was used as secondary antibody for 30 minutes at room temperature. The protein detection was performed using enhanced chemiluminescent (ECL) detection system.

\section{Statistical analysis}

The all performed experiments were repeated for three times, and obtained data were presented as mean \pm standard deviation (SD). The Student's t-test, Tukey (post-hoc), and one-way analysis of variance (ANOVA) were used to statistical analysis using the Graph Pad Prism software. The $p$-value $<0.05$ was considered as significant.

\section{Results}

\section{Cancer cells viability}

The GC cell line AGS showed a time and concentration dependent decrease in cell viability following treatment with hWJSC-CM $(38,40,42,44$, and $46 \%)$ and hWJSC-CL $(11,12,13,14$, and $15 \%)$ at $24-72$ hours. The viability of cancer cells significantly decreased in high concentrations of hWJSC-CM and hWJSC-CL as compared with untreated controls. However, treatment of cancer cells with hWJSC-CL showed statistically significant decreases in cancer cell viability compared to hWJSC-CM. The half maximal inhibitory concentration $\left(\mathrm{IC}_{50}\right)$ of hWJSC-CM and hWJSC-CL on GC cell line AGS cells after 48 hours were $44 \%$ and $14 \%$, respectively (Figure 1 ). 


\section{Cancer cells migration and invasion}

In the wound-healing migration assay, the GC cells showed significantly decrease in migration following treatment with the hWJSC-CM (44\%) and hWJSC-CL (14\%) as compared with untreated cancer cells (Figure 2). In the transwell invasion assay, the hWJSC-CM (44\%) and hWJSC-CL (14\%) significantly inhibited invasion of GC cells as compared with untreated cancer cells (Figure 3). However, treatment of cancer cells with hWJSC-CL (14\%) showed statistically significant decreases in cancer cell migration and invasion compared to hWJSC-CM (44\%).

\section{Cancer cells apoptosis}

The apoptosis quantification demonstrated that the early and late apoptosis rate of GC cell line significantly increased following treatment with hWJSC-CM (44\%) and hWJSC-CL (14\%). However, treatment of cancer cells with hWJSC-CL (14\%) showed statistically significant increase in apoptosis rate of cancer cells compared to hWJSC-CM (44\%) (Figure 4). The evaluation of apoptosis-related genes mRNA expression demonstrated that the hWJSC-CM (44\%) and hWJSC-CL (14\%) significantly increased mRNA expression of $B A X$ and SMAC genes as well as significantly decreased mRNA expression of BCL2 and SURVIVIN genes. We observed that modification of apoptosis-related genes mRNA expression in GC cells by hWJSC-CL (14\%) was significantly more than hWJSC-CM (44\%) (Figure 5).

\section{MAPK and NF-KB signaling pathways}

To investigate whether hWJSC-CM (44\%) and hWJSC-CL (14\%) change viability, migration, invasion, and apoptosis of GC cell line AGS cells, we investigated the proteins levels involved in MAPK and NF-KB signaling pathways. In the NF-KB signaling pathway, the obtained results demonstrated that the phosphoand total-p65 proteins expression were decreased significantly in the hWJSC-CM and hWJSC-CL treated cancer cells (Figure 6). In the MAPK signaling pathway, the hWJSC-CM and hWJSC-CL were increased the active phospho- and total- p38 and phospho- and total- JNK levels in treated cancer cells. These findings suggested that the NF-KB and MAPK signaling pathways play an important role in anticancer activity of hWJSC-CM and hWJSC-CL in GC cancer cells. However, we observed that modification of MAPK and NFKB signaling pathways in GC cells by hWJSC-CL (14\%) was significantly more than hWJSC-CM (44\%) (Figure 7).

\section{Discussion}

The GC is the third cause of cancer mortality worldwide with a poor prognosis and relatively short survival [11]. Despite the great progress in therapeutic methods, prognosis of GC patients remains poor, due to frequent metastasis, drug resistance, and relapse of GC [12]. Therefore, further studies on critical mechanisms in GC progress are imperative. The MSCs including the umbilical cord hWJSCs have been 
recently used as anticancer agents several human malignancies $[8,13]$. The hWJSCs harvest painlessly from human umbilical cord which are usually discarded, highly proliferative, widely multipotent, and have long telomeres [14]. The hWJSCs are potentially quite applicable to human patients without a complete genetic match since they are unlikely to induce an acute inflammatory response [15].

The uncontrolled proliferation and high viability of different human cells as well as metastasis to adjacent tissues are the most important cause of cancer progression and mortality $[16,17]$. In the present study, we evaluated viability, proliferation, migration, and invasion of GC cell line AGS treated with hWJSC-CM and hWJSC-CL, as compared untreated controls. The obtained results demonstrated that the hWJSC significantly decreased the GC cells viability, proliferation, migration, and invasion in a time and concentration dependent manner. In this regards, the previous studies reported that the hWJSC inhibits viability, proliferation, migration, and invasion of various cancer cells $[7,14]$. In a study by Kalamegam et al. reported that the hWJSC-CM and hWJSC-CL suppress cellular growth, proliferation, viability, and migration of ovarian cancer cells through several mechanisms [13]. However, the underlying mechanisms of cancer cells death in the presence of umbilical cord hWJSC remains unknown. Therefore, identification of various underlying anticancer mechanisms of hWJSCs against cancer cells is one of the main objective in several previous studies $[8,13]$.

The inhibition of apoptosis is one of the most important pathological processes in progress of various cancers. Thus, induction of apoptosis is a crucial approach for increase cancer cells death following treatment with various anticancer agents $[18,19]$. In the present study, the effects of hWJSC-CM and hWJSC-CL was evaluated on apoptosis of GC cell line AGS using Annexin V-FITC/PI. The obtained results demonstrated that the apoptosis rates of GC cells significantly increased following treatment with hWJSC-CM and hWJSC-CL. In this regards, the previous studies reported that hWJSC-CM and hWJSC-CL cause to induction of apoptosis in several cancer cells $[7,14]$. In a study by Elias et al. reported that the hWJSCs increased apoptosis rate in the squamous cell carcinoma cell line HEP-2 [20].

The apoptosis process in human is regulated trough interaction of several genes family members [21]. The high expression of BAX and SMAC genes as well as low expression of BCL2 and SURVIVIN genes causes activation of apoptosis onset $[22,23]$. In this regards, our study demonstrated that the hWJSC-CM and hWJSC-CL significantly upregulated mRNA expression of $B A X$ and SMAC genes (as pro-apoptotic genes) as well as downregulated mRNA expression BCL2 and SURVIVIN genes (as anti-apoptotic protein) in GC cells. In a study by Ghorbani and Maleki reported that the hWJSCs induces apoptosis through upregulation and down-regulation of $B A X$ and $B C L 2$ genes expression, respectively [24]. In another study by Elias et al. reported that the hWJSCs up-regulated and down-regulated mRNA expression of P53 and BCL2 genes that leads to induction of apoptosis [20]. These evidences suggested that the hWJSCs induces apoptosis pathway in various cancer cells through modification and regulation of apoptosisrelated genes expression.

The MAPK signaling pathway play an important role in viability, proliferation, metastasis, and invasion of cancer cells [25]. The inhibition of MAPK signaling pathway decrease efficiency of various anticancer 
agents and therapeutic methods [26]. The present study demonstrated that the MAPK signaling pathway was activated by hWJSC-CM and hWJSC-CL in GC cells. On the other hands, the NF-KB signaling pathway play a crucial role in cancer cells proliferation, viability, and carcinogenesis [27]. The inhibition of the NF-KB signaling pathway induces apoptosis and inhibits invasion and migration in various cancer cells [28]. In the present study, we showed that the hWJSC-CM and hWJSC-CL significantly inhibits the NF-KB signaling pathway in the GC cells. These results suggested that the MAPK and NF-KB signaling pathway may be involved in inhibitory effects of hWJSC-CM and hWJSC-CL against proliferation, viability, migration, and invasion of GC cells.

\section{Conclusions}

In general, our study presents a novel therapeutic approach to treatment of patients with GC. Our study showed that the hWJSC-CM and hWJSC-CL inhibits the viability, proliferation, migration, and invasion of GC cell line AGS through several molecular mechanisms. The hWJSC-CM and hWJSC-CL induces apoptosis in GC cells through modification of apoptosis-related genes expression. Moreover, the NF-KB, MAPK signaling pathways may be involved in inhibitory effects of hWJSC-CM and hWJSC-CL in GC cells. Therefore, the hWJSC can be used in future to control and even treatment of patients with GC. However, further studies are required to identification the exact effects of hWJSCs on physiopathology of GC.

\section{Declarations}

\section{Acknowledgements}

None.

\section{Author Contributions}

All authors had an equal role in design, work, statistical analysis, and manuscript writing.

\section{Ethical approval}

All procedures performed in studies involving human participants were in accordance with the ethical standards of the institutional research committee and with the 1964 Helsinki declaration and its later amendments or comparable ethical standards.

\section{Informed consent}

Informed consent was obtained from all individual participants included in the study. 


\section{Conflict of interest}

The authors declare no conflict of interest.

\section{Consent to participate}

Patients already gave their consent to participate in the corresponding trials.

\section{Consent for publication}

All authors have consented to submission of this article for publication.

\section{Role of the funding source}

None.

\section{Data availability}

Not generally available

\section{References}

1. Fitzmaurice C, Dicker D, Pain A, Hamavid H, Moradi-Lakeh M, Maclntyre MF, Allen C, Hansen G, Woodbrook R, Wolfe C, Hamadeh RR. The global burden of cancer 2013. JAMA Oncol 2015;1(4):50527.

2. Inoue M. Tsugane. Epidemiology of gastric cancer in Japan. Postgrad Med J 2005;81(957):419-24.

3. Taheri E, Ghorbani S, Safi M, Sani NS, Amoodizaj FF, Hajazimian S, Heidari M, Isazadeh A, Heidari M. Inhibition of Colorectal Cancer Cell Line CaCo-2 by Essential Oil of Eucalyptus camaldulensis Through Induction of Apoptosis. Acta Med Iran 2020;58(6):260-265.

4. Firouzi Amoodizaj F, Baghaeifar S, Taheri E, Farhoudi Sefidan Jadid M, Safi M, Seyyed Sani N, Hajazimian S, Isazadeh A, Shanehbandi D. Enhanced anticancer potency of doxorubicin in combination with curcumin in gastric adenocarcinoma. J Biochem Mol Toxicol 2020;e22486.

5. Fong CY, Chak LL, Biswas A, Tan JH, Gauthaman K, Chan WK, Bongso A. Human Wharton's jelly stem cells have unique transcriptome profiles compared to human embryonic stem cells and other 
mesenchymal stem cells. Stem Cell Rev Rep 2011;7(1):1-6.

6. Fong CY, Subramanian A, Biswas A, Gauthaman K, Srikanth P, Hande MP, Bongso A. Derivation efficiency, cell proliferation, freeze-thaw survival, stem-cell properties and differentiation of human Wharton's jelly stem cells. Reprod Biomed Online 2010;21(3):391-401.

7. Gauthaman K, Fong CY, Suganya CA, Subramanian A, Biswas A, Choolani M, Bongso A. Extraembryonic human Wharton's jelly stem cells do not induce tumorigenesis, unlike human embryonic stem cells. Reprod Biomed Online 2012.24(2):235-46.

8. Hajazimian S, Maleki M, Mehrabad SD, Isazadeh A. Human Wharton's jelly stem cells inhibit endometriosis through apoptosis induction. Reproduction 2020;159(5):549-58.

9. Ahn JO, Coh YR, Lee HW, Shin IS, Kang SK, Youn HY. Human adipose tissue-derived mesenchymal stem cells inhibit melanoma growth in vitro and in vivo. Anticancer Res 2015;35(1):159-68.

10. Hendijani F, Javanmard SH. Dual protective and cytotoxic benefits of mesenchymal stem cell therapy in combination with chemotherapy/radiotherapy for cancer patients. Crit Rev Eukaryot Gene Expr 2015;25(3):203-207.

11. Torre LA, Bray F, Siegel RL, Ferlay J, Lortet-Tieulent J, Jemal A. Global cancer statistics, 2012. CA Cancer J Clin 2015;65(2):87-108.

12. Takahashi T, Saikawa Y, Kitagawa Y. Gastric cancer: current status of diagnosis and treatment. Cancers 2013;5(1):48-63.

13. Kalamegam G, Sait KH, Ahmed F, Kadam R, Pushparaj PN, Anfinan N, Rasool M, Jamal MS, AbuElmagd M, Al-Qahtani M. Human Wharton's Jelly Stem Cell (hWJSC) Extracts Inhibit Ovarian Cancer Cell Lines OVCAR3 and SKOV3 in vitro by Inducing Cell Cycle Arrest and Apoptosis. Front Oncol 2018;8:592.

14. Gauthaman K, Fong CY, Arularasu S, Subramanian A, Biswas A, Choolani M, Bongso A. Human Wharton's jelly stem cell conditioned medium and cell-free lysate inhibit human osteosarcoma and mammary carcinoma cell growth in vitro and in xenograft mice. J Cell Biochem 2013;114(2):366-77.

15. Weiss ML, Anderson C, Medicetty S, Seshareddy KB, Weiss RJ, VanderWerff I, Troyer D, Mclntosh KR. Immune properties of human umbilical cord Wharton's jelly-derived cells. Stem Cells 2008;26(11):2865-74.

16. Soheilyfar S, Velashjerdi Z, Hajizadeh YS, Maroufi NF, Amini Z, Khorrami A, Azimian SH, Isazadeh A, Taefehshokr S, Taefehshokr N. In vivo and in vitro impact of miR-31 and miR-143 on the suppression of metastasis and invasion in breast cancer. J BUON 2018;23(5):1290-6.

17. Maroufi NF, Vahedian V, Hemati S, Rashidi M, Akbarzadeh M, Zahedi M, Pouremamali F, Isazadeh A, Taefehshokr S, Hajazimian S, Seraji N. Targeting cancer stem cells by melatonin: Effective therapy for cancer treatment. Pathol Res Pract 2020;152919.

18. Maroufi NF, Vahedian V, Akbarzadeh M, Mohammadian M, Zahedi M, Isazadeh A, Pouremamali F, Taefehshokr S, Heidari M, Rashidi M, Nouri M. The apatinib inhibits breast cancer cell line MDA-MB231 in vitro by inducing apoptosis, cell cycle arrest, and regulating nuclear factor-KB (NF-кB) and mitogen-activated protein kinase (MAPK) signaling pathways. Breast Cancer 2020;1-8. 
19. Taheri E, Ghorbani S, Safi M, Seyyed Sani N, Firouzi Amoodizaj F, Heidari M, Chavoshi R, Hajazimian S, Isazadeh A, Heidari M. Inhibition of Colorectal Cancer Cell Line CaCo-2 by Essential Oil of Eucalyptus Camaldulensis Through Induction of Apoptosis. Acta Med Iran 2020;58(6):260-265.

20. Elias WY, Ayoub MS, Malahy HE. The effect of Wharton's jelly mesenchymal stem cells on a squamous cell carcinoma cell line. Arch Cancer Res 2016;4:1.

21. Brunelle JK, Letai A. Control of mitochondrial apoptosis by the Bcl-2 family. J Cell Sci 2009;122(4):437-41.

22. Huerta S, Heinzerling JH, Anguiano-Hernandez YM, Huerta-Yepez S, Lin J, Chen D, Bonavida B, Livingston EH. Modification of gene products involved in resistance to apoptosis in metastatic colon cancer cells: Roles of Fas, Apaf-1, NFkB, IAPs, Smac/DIABLO, and AIF. J Surg Res 2007;142(1):18494.

23. Chelluboina B, Klopfenstein JD, Gujrati M, Rao JS, Veeravalli KK. Temporal regulation of apoptotic and anti-apoptotic molecules after middle cerebral artery occlusion followed by reperfusion. Mol Neurobiol 2014;49(1):50-65.

24. Ghorbani S, Maleki M. Inhibition of Cervical Cancer Cell Line Hela by Human Wharton's Jelly Stem Cells Through Induction of Apoptosis. Gene Cell Tissue 2020;7(1):e99206.

25. Kim EK, Choi EJ. Pathological roles of MAPK signaling pathways in human diseases. Biochim Biophys Acta Mol Basis Dis 2010;1802(4):396-405.

26. Cao J, Tong C, Liu Y, Wang J, Ni X, Xiong MM. Ginkgetin inhibits growth of breast carcinoma via regulating MAPKs pathway. Biomed Pharmacother 2017;96:450-8.

27. Hoesel B, Schmid JA. The complexity of NF-KB signaling in inflammation and cancer. Mol Cancer 2013;12(1):1-5.

28. Wang G, Li J, Zhang L, Huang S, Zhao X, Zhao X. Celecoxib induced apoptosis against different breast cancer cell lines by down-regulated NF-KB pathway. Biochem Biophys Res Commun 2017;490(3):969-76.

\section{Table}

Table 1. The sequences and characteristics of used primers for quantification of genes mRNA expression. 


\begin{tabular}{|c|c|c|c|}
\hline Genes & Primers sequences & $\mathrm{T}_{\mathrm{m}}$ & Products size \\
\hline \multirow[t]{2}{*}{$B A X$} & F-CCCGAGAGGTCTTTTTCCGAG & $60^{\circ} \mathrm{C}$ & \multirow[t]{2}{*}{155 bp } \\
\hline & R-CCAGCCCATGATGGTTCTGAT & $60^{\circ} \mathrm{C}$ & \\
\hline \multirow[t]{2}{*}{$B C L 2$} & F-GATGGGATCGTTGCCTTATG & $56^{\circ} \mathrm{C}$ & \multirow[t]{2}{*}{$223 \mathrm{bp}$} \\
\hline & R-GCGGAACACTTGATTCTGG & $56^{\circ} \mathrm{C}$ & \\
\hline \multirow[t]{2}{*}{$S M A C$} & F-CAGAGGAGGAAGATGAAGTGTG & $55^{\circ} \mathrm{C}$ & \multirow[t]{2}{*}{196 bp } \\
\hline & R-GCGGTTATAGAGGCCTGATCTG & $56^{\circ} \mathrm{C}$ & \\
\hline \multirow[t]{2}{*}{ SURVIVIN } & F-CCСTTTCTCAAGGACCACCG & $52^{\circ} \mathrm{C}$ & \multirow[t]{2}{*}{172 bp } \\
\hline & R-GTTCCTCTATGGGGTCGTCA & $51^{\circ} \mathrm{C}$ & \\
\hline \multirow[t]{2}{*}{ ACTIN } & F-AGAGCTACGAGCTGCCTGAC & $61^{\circ} \mathrm{C}$ & \multirow[t]{2}{*}{186 bp } \\
\hline & R-AGCACTGTGTTGGCGTACAG & $59^{\circ} \mathrm{C}$ & \\
\hline
\end{tabular}

\section{Figures}
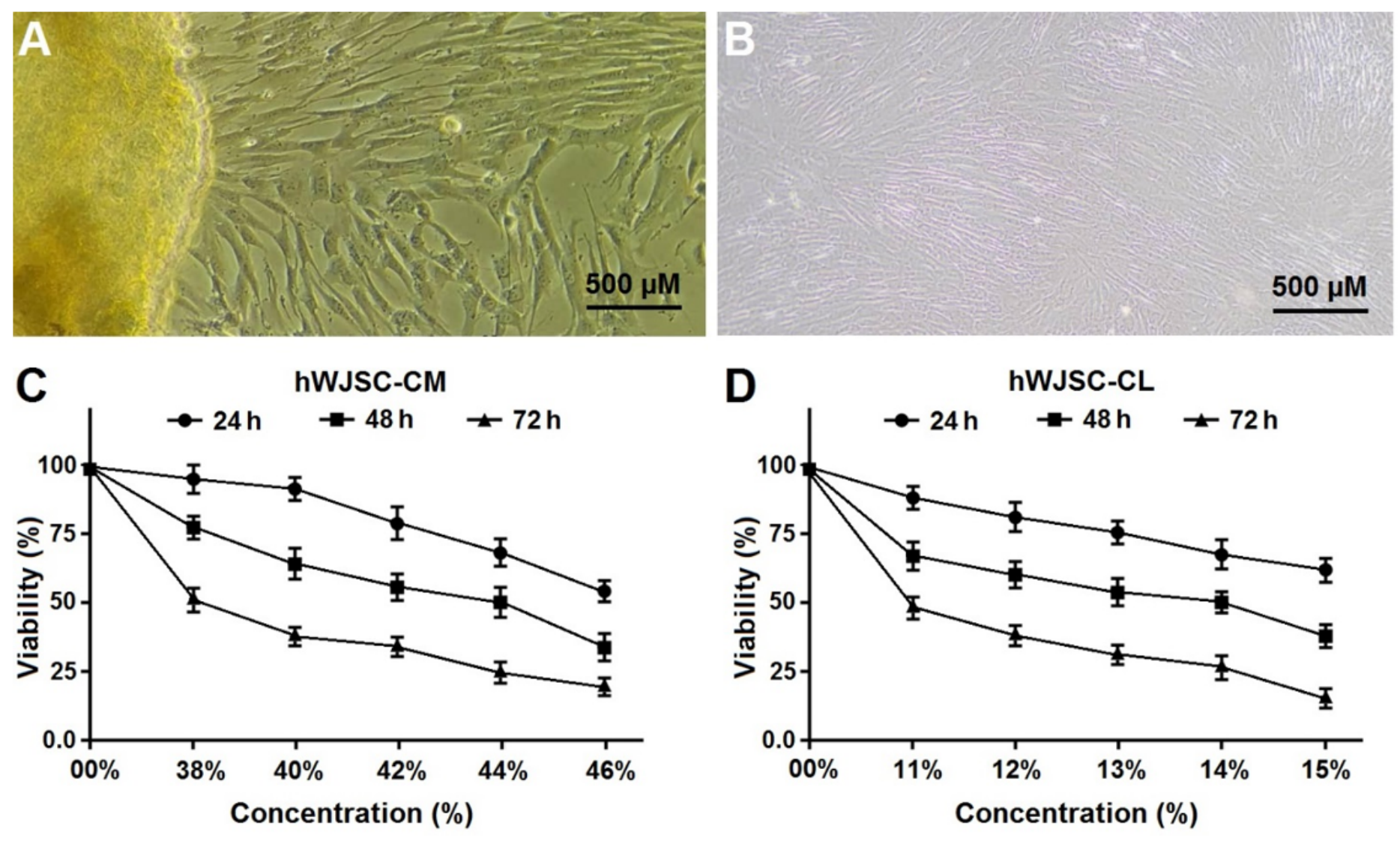

Figure 1 
The primary cultured hWJSCs with fibroblasts characteristic in early passages (A) and long fibroblasts characteristic in later passages (B). The viability of GC cell line AGS treated with hWJSC-CM (C) and hWJSC-CL (D) at 24,48 , and 72 hours. The anticancer activity of hWJSC-CL was significantly more than hWJSC-CM.
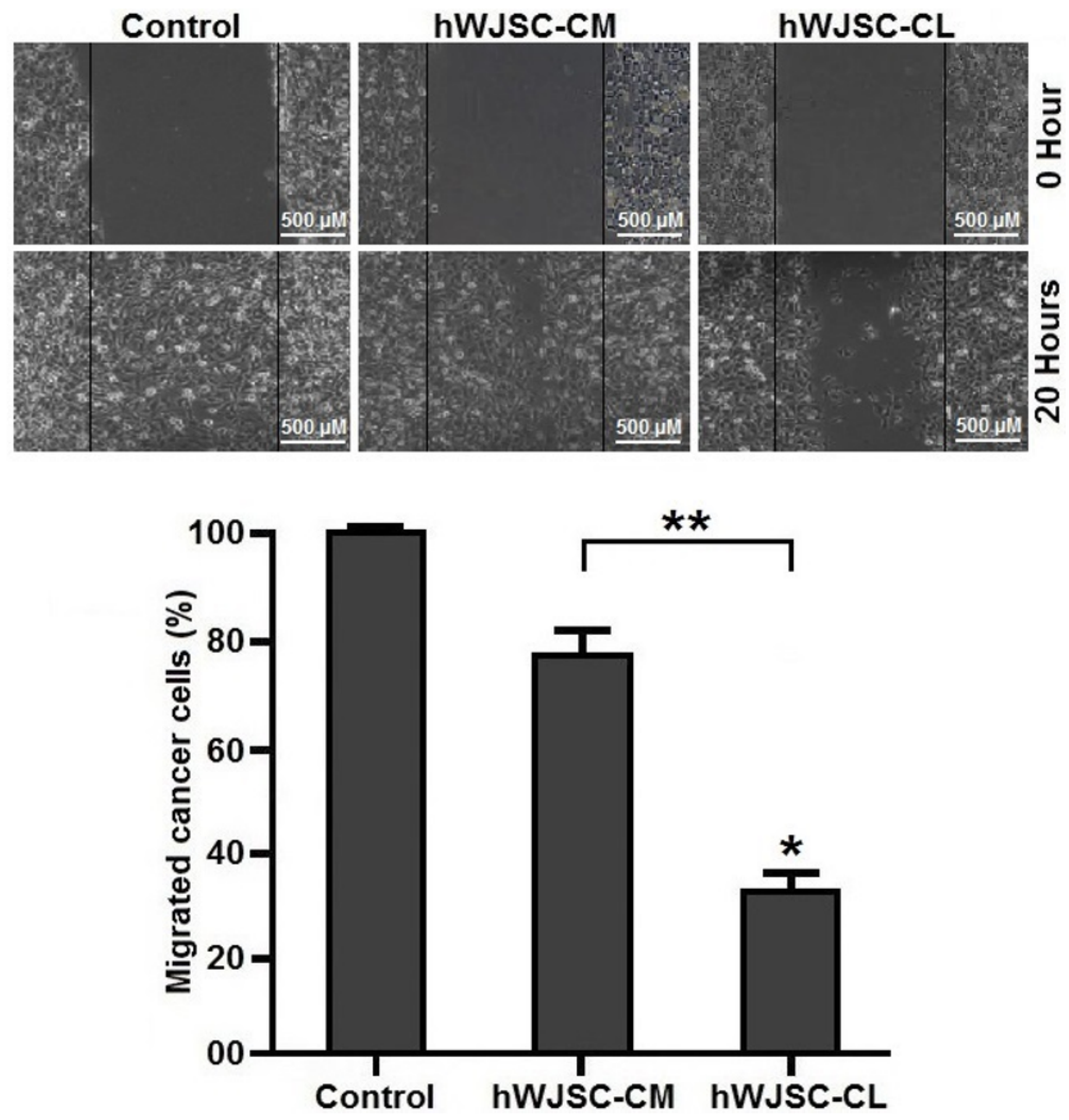

Figure 2 
The GC cell line AGS migration evaluated with wound healing migration assay following treatment with hWJSC-CM (44\%) and hWJSC-CL (14\%) for 10 to 20 hours. The inhibition of migration in treated cancer cells with hWJSC-CL was significantly more than hWJSC-CM.

Control

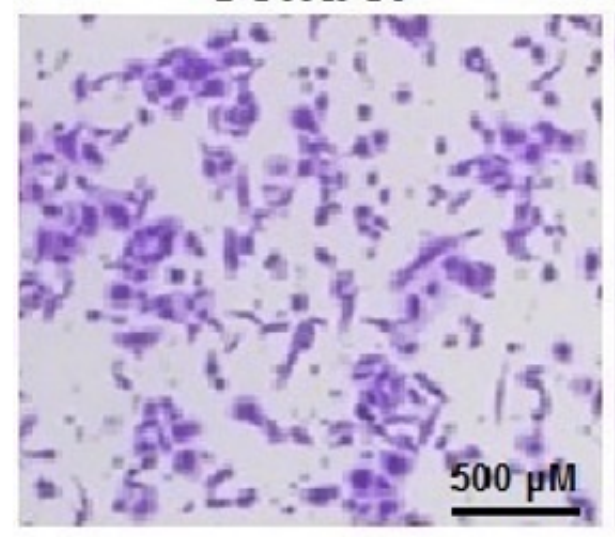

hWJSC-CM

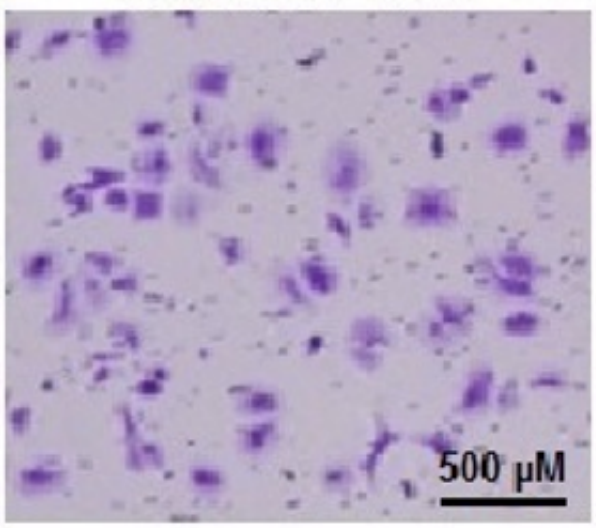

hWJSC-CL

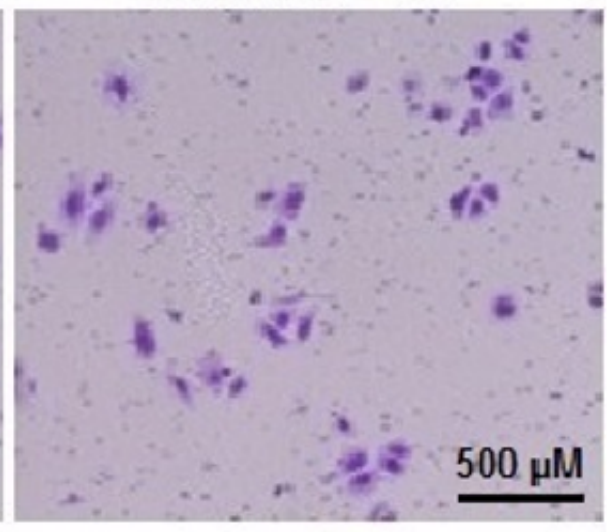

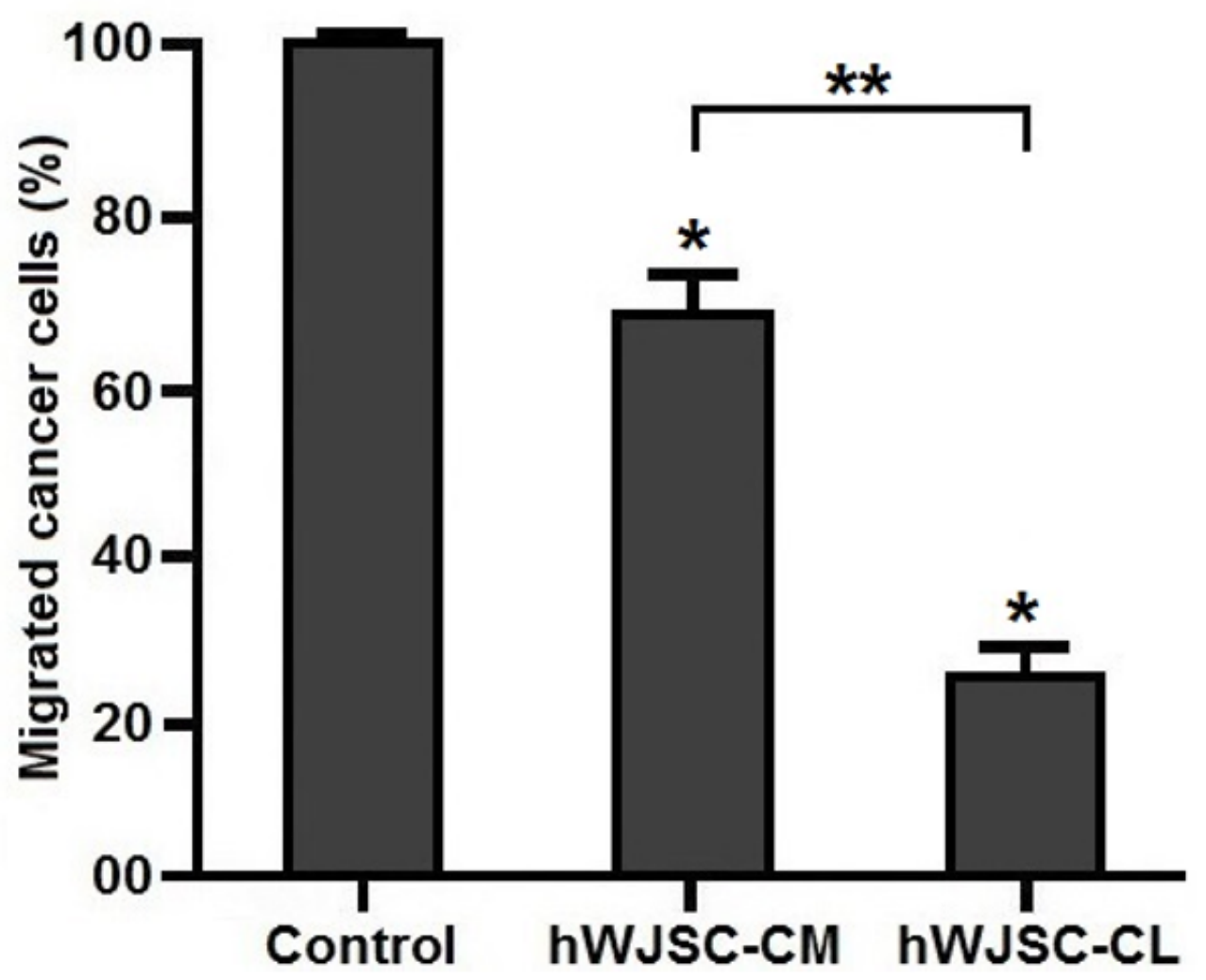

Figure 3

The GC cell line AGS invasion evaluated with transwell invasion assay following treatment with hWJSCCM (44\%) and hWJSC-CL (14\%) for 48 hours. The inhibition of invasion in treated cancer cells with hWJSC-CL was significantly more than hWJSC-CM. 

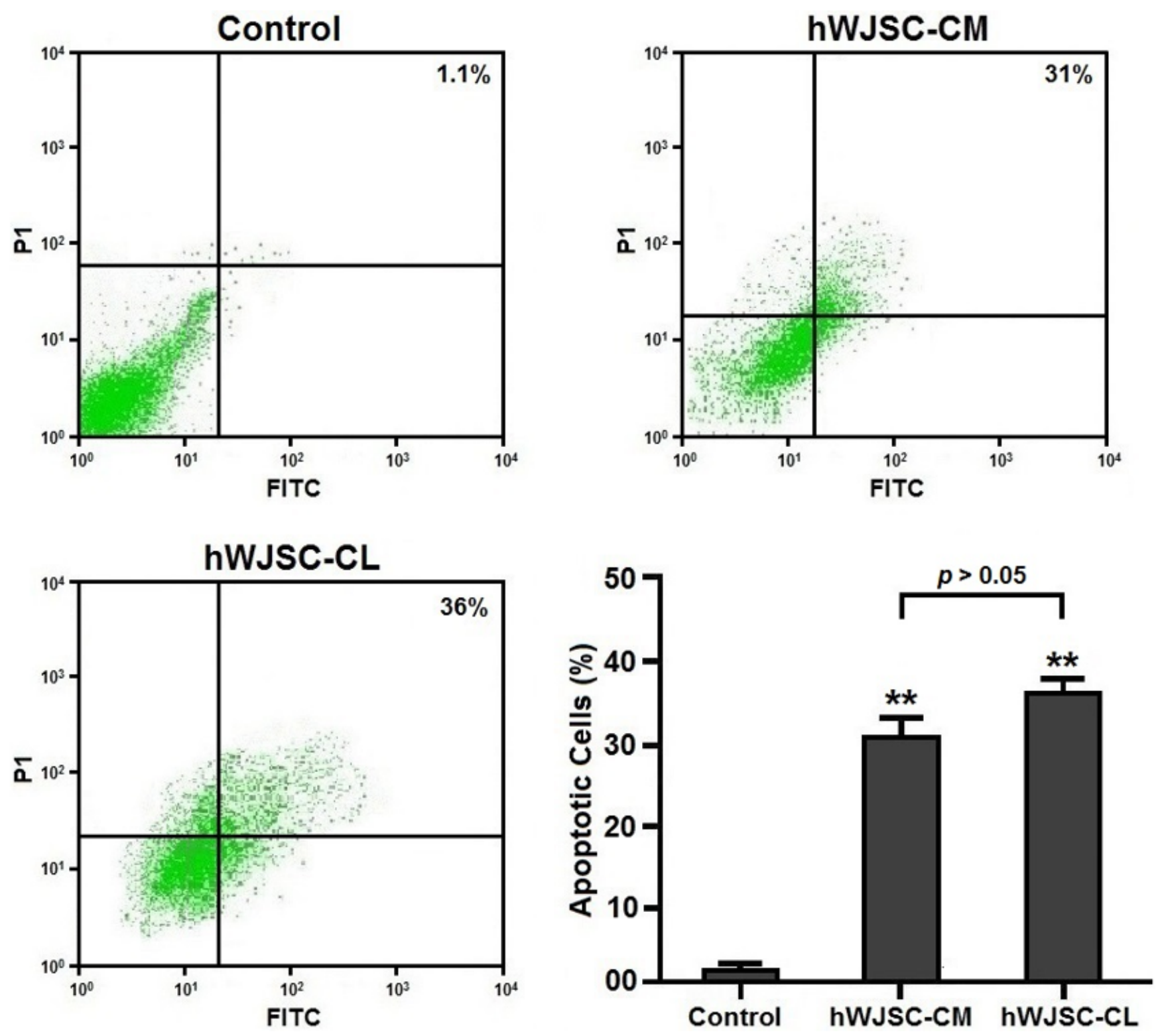

Figure 4

The GC cell line AGS apoptosis evaluated with annexin V-FITC/PI assay following treatment with hWJSCCM (44\%) and hWJSC-CL (14\%) for 48 hours. The induction of apoptosis in the cancer cells treated with hWJSC-CL was significantly more as compared with hWJSC-CM. 

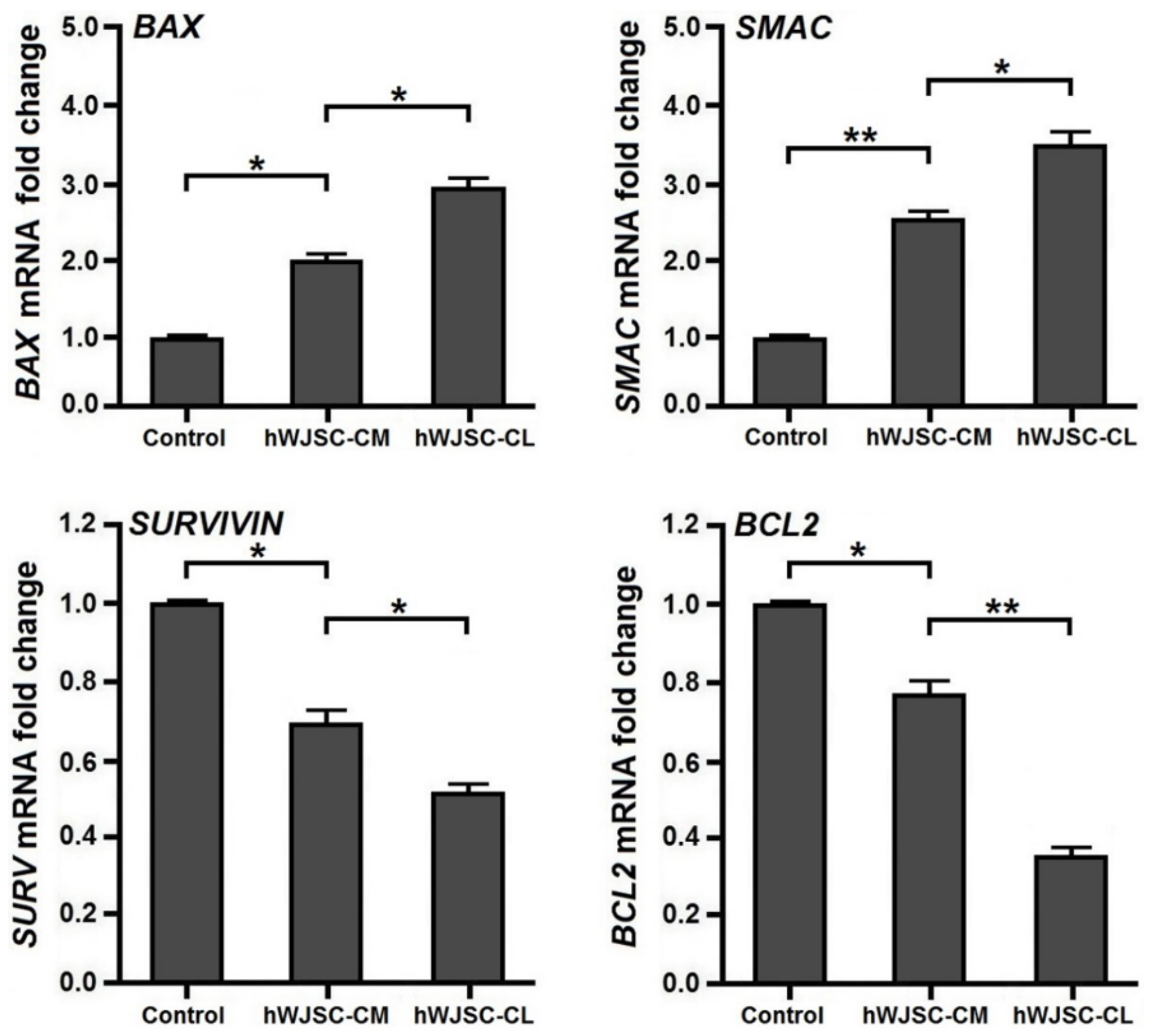

Figure 5

The mRNA expression analysis of apoptosis related BAX, SMAC, SURVIVIN, and BCL-2 genes by qRT-PCR following treatment with hWJSC-CM (44\%) and hWJSC-CL (14\%) for 48 hours. The modification of apoptosis related genes mRNA expression in the cancer cells treated with hWJSC-CL was significantly more as compared with hWJSC-CM. 

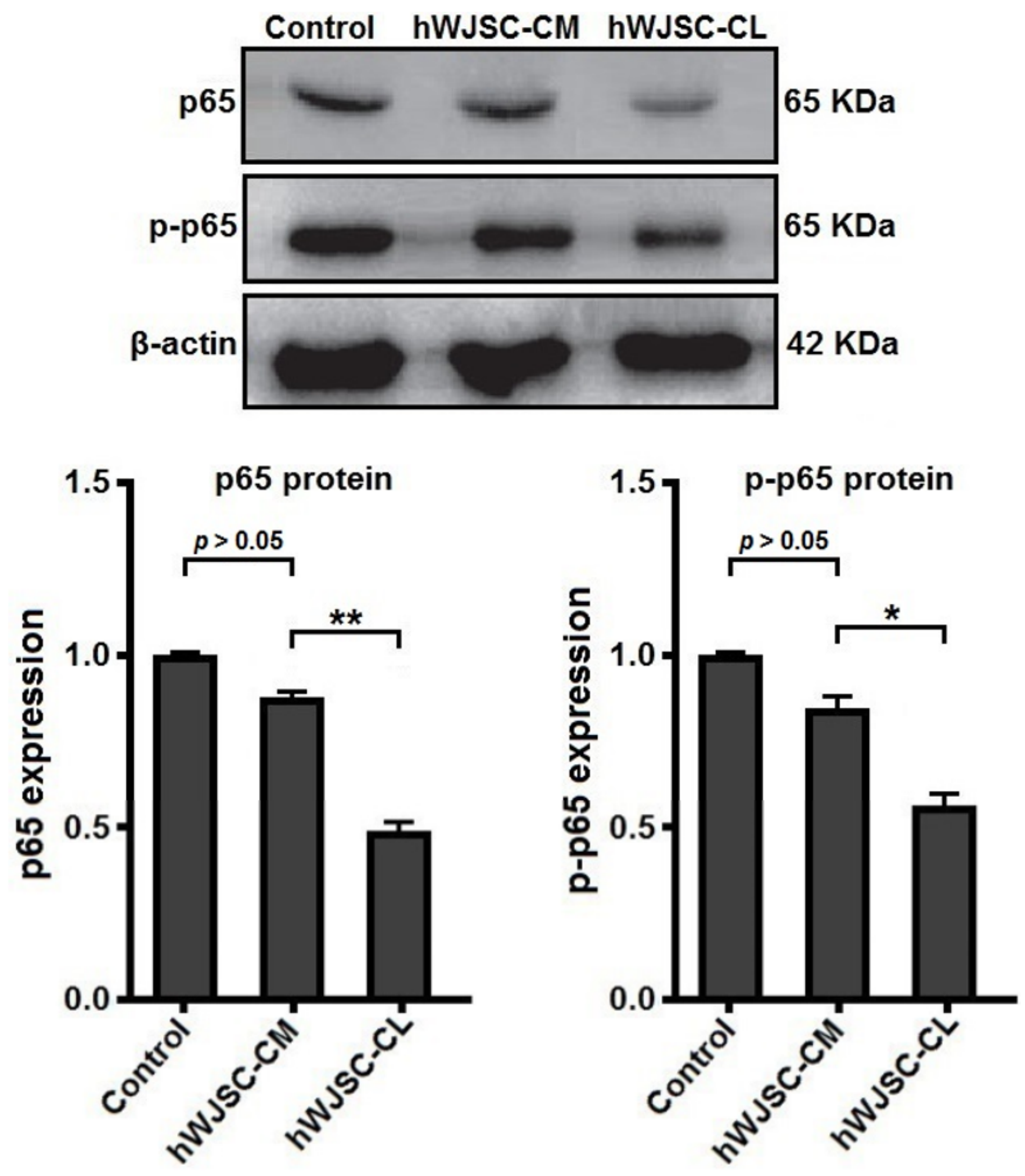

Figure 6

The expression of proteins involved in NF-KB signaling pathway evaluated using western blotting analysis following treatment with hWJSC-CM (44\%) and hWJSC-CL (14\%) for 48 hours. The modification of NF-KB in the cancer cells treated with hWJSC-CL was significantly more as compared with hWJSC-CM. 

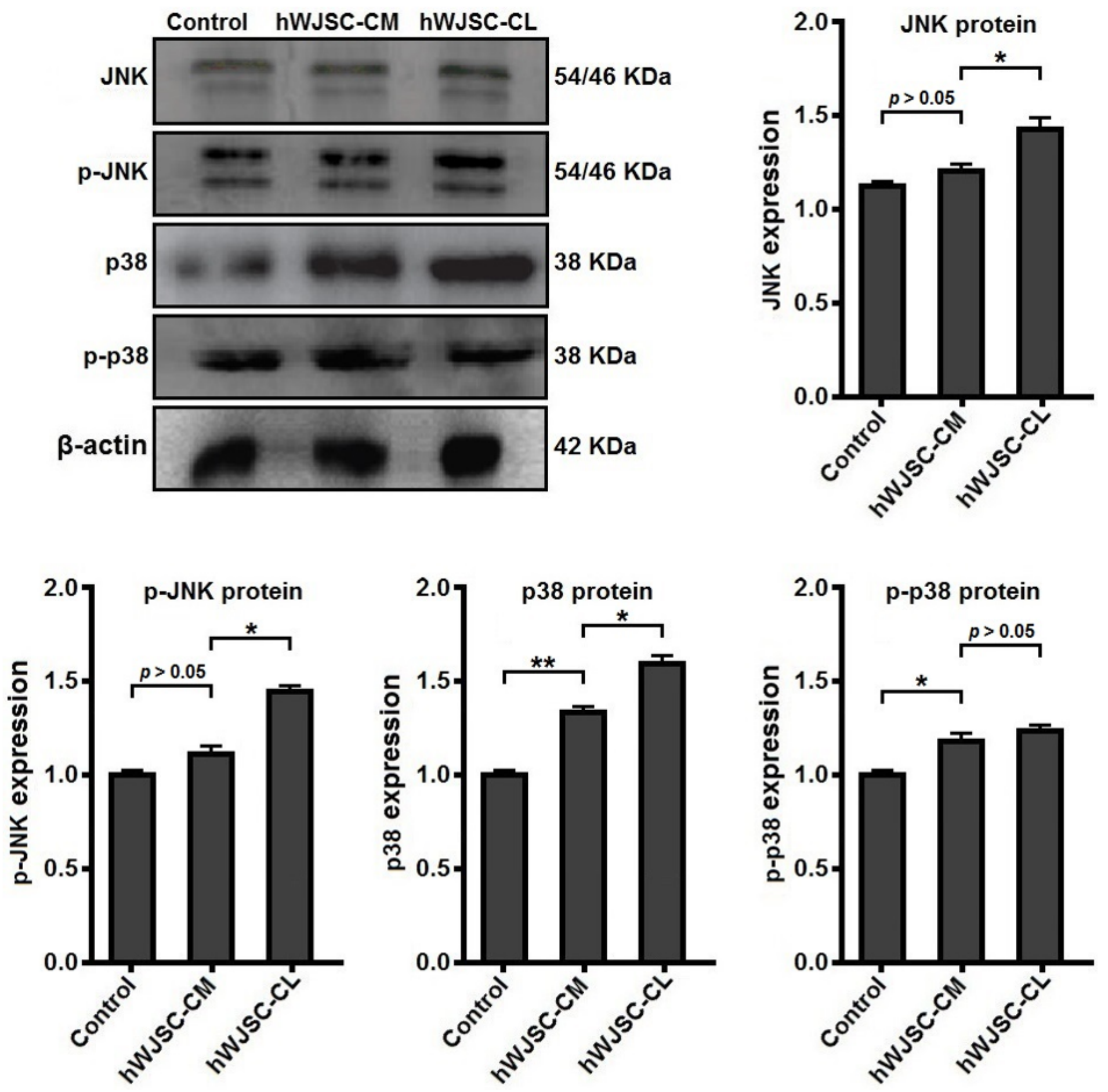

Figure 7

The expression of proteins involved in MAPK signaling pathways evaluated using western blotting analysis following treatment with hWJSC-CM (44\%) and hWJSC-CL (14\%) for 48 hours. The modification of MAPK in the cancer cells treated with hWJSC-CL was significantly more as compared with hWJSC-CM. 